\title{
A Novel Mutation in the ROGDI Gene in a Patient with Kohlschütter-Tönz Syndrome
}

\author{
Mathilde Huckert ${ }^{\mathrm{a}-\mathrm{c}}$ Helen Mecili ${ }^{\mathrm{d}}$ Virginie Laugel-Haushalter ${ }^{\mathrm{h}}$ \\ Corinne Stoetzel $^{c}$ Jean Muller ${ }^{\mathrm{e}, \mathrm{f}, \mathrm{h}} \quad$ Elisabeth Florig ${ }^{\mathrm{V}}$ Vincent Laugel $^{\mathrm{d}}$ \\ Marie-Cécile Manière ${ }^{a, b}$ Hélène Dollfus ${ }^{c}$ Agnès Bloch-Zupan ${ }^{a, b, h}$ \\ ${ }^{a}$ Faculty of Dentistry, University of Strasbourg (UdS), ${ }^{\text {b }}$ Reference Center for Orodental Manifestations of Rare \\ Diseases, Pôle de Médecine et Chirurgie Bucco-dentaires, Hôpital Civil, Hôpitaux Universitaires de Strasbourg \\ (HUS), ' Laboratoire de Génétique Médicale, INSERM UMR_S 1112, Strasbourg Medical School, UdS, dCentre de \\ Référence des Maladies Neuromusculaires d'Origine Génétique de l'Enfant et l'Adulte, Hôpital de Hautepierre, \\ HUS, e'Laboratoire de Diagnostic Génétique, HUS, ${ }^{f}$ ICube UMR 7357, UdS, CNRS, LBGI, and ${ }^{9}$ Laboratoire de \\ Cytogénétique, Cytologie et Histologie Quantitative, Hôpital de Hautepierre, HUS, Strasbourg, and ${ }^{\mathrm{h}}$ Institut de \\ Génétique et de Biologie Moléculaire et Cellulaire (IGBMC), UMR 7104 and INSERM U964, UdS, Illkirch, France
}

\section{Key Words}

Amelogenesis imperfecta $\cdot$ Epilepsy $\cdot$ Kohlschütter-Tönz syndrome $\cdot$ Leucine zipper domain · Mutations $\cdot$ ROGDI

\footnotetext{
Abstract

Kohlschütter-Tönz Syndrome (KTZS) is an autosomal recessive disorder caused by mutations in the ROGDI gene. This syndrome is characterized by epilepsy, psychomotor regression and amelogenesis imperfecta. In this paper, we report a case of a 13-year-old Malian girl presenting with this rare disease. By genetic analysis, we identified a novel ROGDI homozygous mutation NM_024589.1: c.117+1G>T [Chr16 (GRCh37): g.4852382C >A] which confirmed the diagnosis of Kohlschütter-Tönz syndrome. The mutation abolishes the usual splice donor site of intron 2 which leads to the deletion of exon 2 and in-frame assembly of exon 3. Exon 2 encodes a highly conserved leucine-rich region that is essential for ROGDI protein function. Hence, this deletion may affect the function of the ROGDI protein.

(c) 2014 S. Karger AG, Basel
}

Kohlschütter-Tönz syndrome (KTZS, OMIM 226750) is a rare autosomal recessive syndrome characterized by epilepsy, psychomotor regression and amelogenesis imperfecta (AI) due to mutations in the ROGDI gene in 16p13.3 [Mory et al., 2012; Schossig et al., 2012a].

$\mathrm{AI}$ is a heterogeneous group of developmental diseases that affect the formation, structure and clinical appearance of enamel. AI can be classified into different subtypes (hypoplastic, hypomineralized, and hypomature) based on the nature of the enamel defect. The prevalence of AI varies from $1 / 700$ to $1 / 14,000$, and it can be inherited in an autosomal dominant, autosomal recessive or X-linked manner [Aldred et al., 2003; Crawford et al., 2007; Bloch-Zupan et al., 2012; Gadhia et al., 2012]. AI can present as an isolated disease or coexist with other abnormalities and symptoms as part of syndromic disorders such as KTZS.

Since the first case of KTZS reported in 1974 [Kohlschütter et al., 1974], around 20 cases have been published so far delineating a similar phenotype. Several

\section{KARGER}

E-Mail karger@karger.com

www.karger.com/msy (c) 2014 S. Karger AG, Basel

$1661-8769 / 14 / 0056-0293 \$ 39.50 / 0$ 


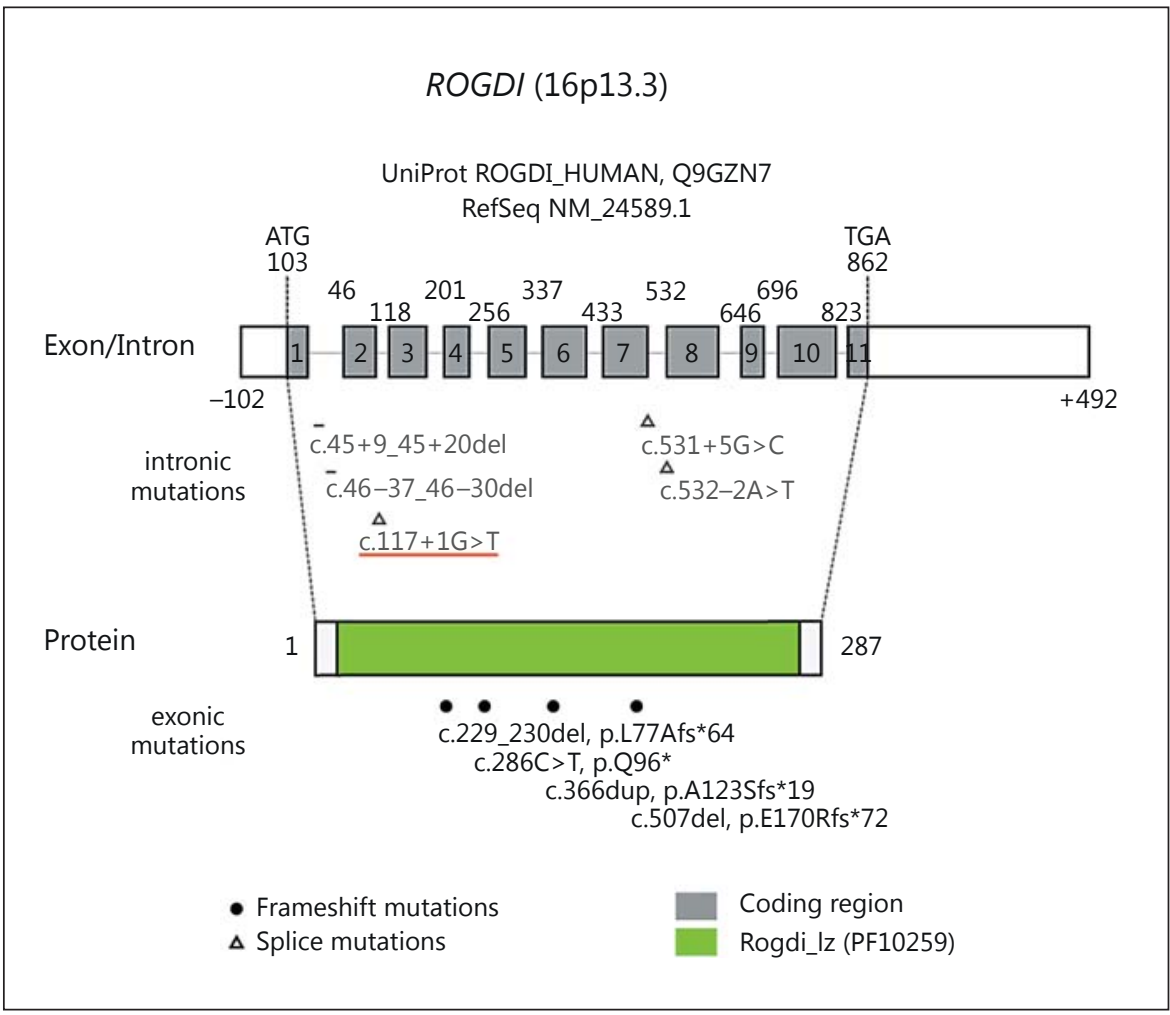

Fig. 1. ROGDI exonic map. Locations of the different mutations described in the literature are shown. The new mutation c. $117+1 \mathrm{G}>\mathrm{T}$ is underlined in red.

mutations in the ROGDI gene were described since 2012 in European KTZS patients (fig. 1) [Mory et al., 2012, 2014; Schossig et al., 2012a, b; Tucci et al., 2013].

We report the case of a 13-year-old girl, the third child of consanguineous parents from Mali, who presented with hypomineralized/hypomature AI, psychomotor delay and pharmaco-resistant epilepsy. Due to the similarity of this phenotype with KTZS, we investigated the ROGDI gene for potential mutations by Sanger sequencing.

\section{Case Report}

The girl and her parents were recruited and examined clinically by dentists at the Reference Center for Orodental Manifestations of Rare Diseases at the Strasbourg University Hospital. The family consented to participate in the French Ministry National Program for Clinical Research, PHRC 2008 HUS No. 4266 'Amelogenesis imperfecta: clinical and molecular study' (ClinicalTrials.gov Identifier: NCT01746121) and in the Interreg/Offensive Sciences A27 projects. Personal and clinical data were entered into D[4]/Phenodent, a dental defects diagnostic database (www.phenodent.org).

The patient was born at term by emergency caesarean section due to pregnancy-induced hypertension. Weight at birth was 3.1 $\mathrm{kg}$ at 39 weeks and 3 days of amenorrhea (25th percentile of birth). She required resuscitation at birth due to meconium aspiration
(Apgars 3, 8 and 10), and was transferred to the special care unit where she received oxygen and antibiotics for $24 \mathrm{~h}$ before being moved to the transitional care ward.

She presented with seizures starting in her 3rd week of life and manifested by hypotonia, peribuccal cyanosis, and eye rolling. At 1 month she underwent surgery for a strangulated inguinal hernia. Shortly after discharge, she presented to the emergency room with an episode of loss of consciousness, which was attributed to gastroesophageal reflux. Two further events followed at 4 and 5 months; her parents described several additional episodes in which she became hypotonic and unresponsive with signs of central cyanosis. All episodes lasted less than $10 \mathrm{~min}$.

At 1 year of age, after presenting with a clear right-sided partial seizure, she was started on carbamazepine and clonazepam, and the working diagnosis of seizures secondary to suspected hypoxic ischemic encephalopathy was made. The seizures continued despite the use of multiple anti-convulsive agents (topiramate, phenobarbital and vigabatrin) as well as a trial of a ketogenic diet. Physical examination was essentially normal and she continued to grow well along her centile lines. Developmental milestones were delayed: she learned to walk unaided at 19 months and spoke her first words at 3 years.

By 6 years of age, the seizures were occurring on a daily basis, often worse at night and predominantly involving right-sided clonic movements. There was severe developmental delay, with a global functional level of an 18-month-old. There were marked right-sided neglect, ataxic gait and dysmorphic features with frontal bossing, thickened protruding lower lip and genital hypoplasia 


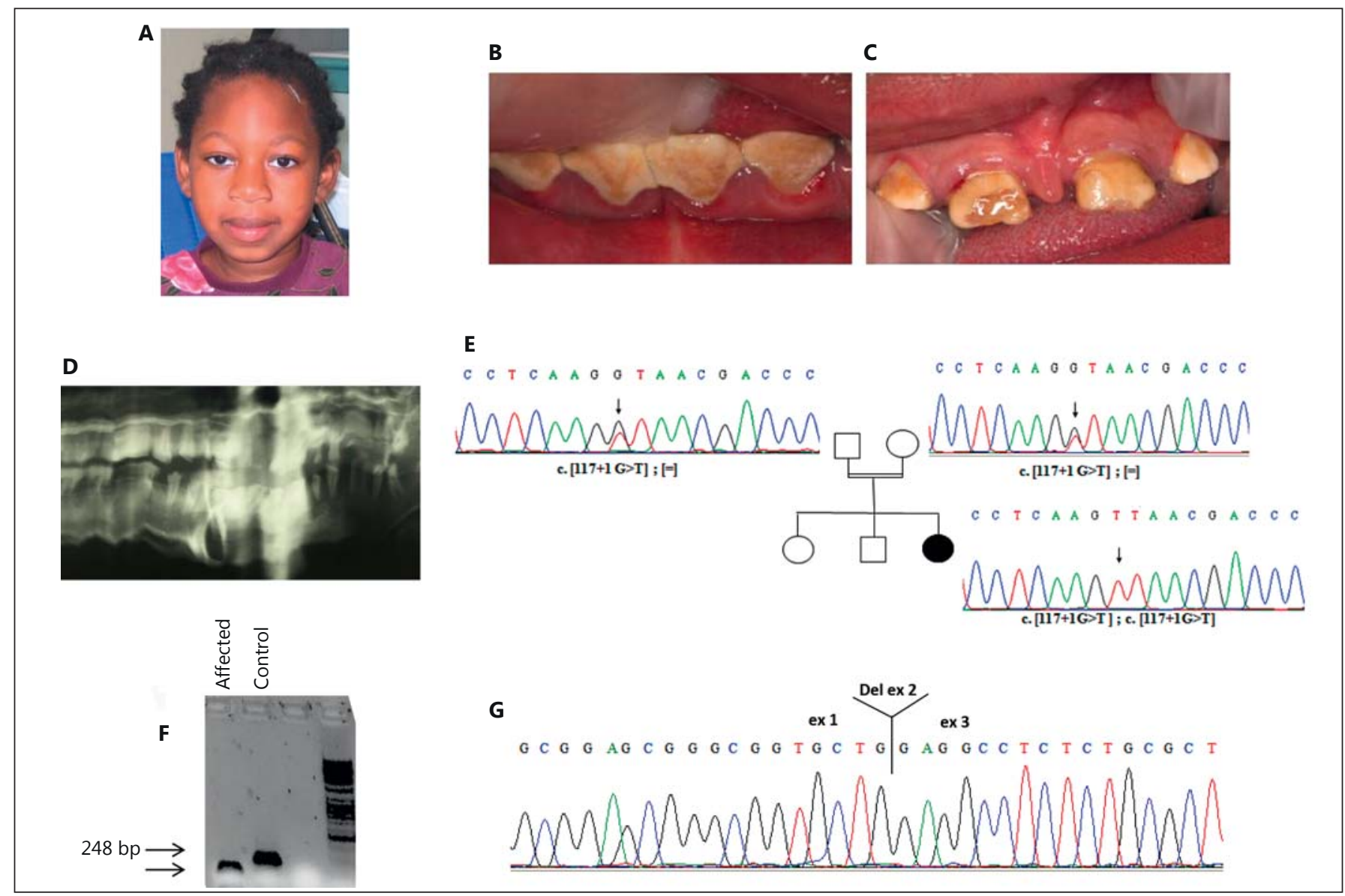

Fig. 2. Phenotype of the affected patient, familial segregation of the mutation and RT-PCR. A Facial photograph of the patient. B Intraoral view of mandibular incisors. C Intraoral view of maxillary incisors. D Panoramic radiograph showing hypomineralized/hypomature AI. The enamel is rough, colored, prone to disintegration, and retains oral biofilm. There is no contrast between dentin and enamel on the radiograph. The poor quality of the panoramic radiograph is due to the behavioral difficulties of the patient, which prevent her from remaining still during the radiographic acquisi- tion procedure. E Identification of a new homozygous mutation c. $117+1 \mathrm{G}>\mathrm{T}$ in the ROGDI gene. The affected proband is homozygous with a $100 \%$ peak. The parents are heterozygous with two $50 \%$ peaks. $[=]=$ normal allele. $\mathbf{F}$ RT-PCR validation of the biological effect of the mutation. The mutation causes the deletion of exon 2 . The amplicon is smaller in the affected patient compared to the control ( $248 \mathrm{bp}$ ). G Sequence of cDNA from the patient confirms the absence of exon 2. (fig. 2A). Head circumference was normal between the 50th and the 75 th percentile. Language was limited to less than 5 words and she exhibited aggressive and impulsive behavior.

The orodental phenotype assessed through oral clinical and radiographic (panoramic radiograph) examinations revealed no abnormalities of tooth number or size, but abnormalities of enamel affecting the primary and permanent dentition were noted (fig. 2B-D). The primary teeth presented severe enamel dysplasia with eruption delay. At 5 years of age, a diagnosis of hypomineralized/hypomature AI was proposed. The enamel of the permanent teeth was rough, colored and retained oral biofilm. Because of her medical and behavioral handicap, dental treatments had to be performed under general anesthesia in 2006, 2007, 2009, and 2012.

\section{Materials and Methods}

DNA Sequencing and Mutation Screening

Saliva samples were collected using the OG-250 Oragene ${ }^{\circledR}$ DNA kit (DNA Genotek Inc., Ottawa, Ont., Canada). Genomic DNA was extracted from saliva according to standard protocols using the prepIT-L2P Oragene ${ }^{\circledR}$ DNA kit (DNA Genotek Inc.). Coding exons and flanking intronic regions of ROGDI were PCR amplified from 50 ng of genomic DNA (online suppl. table 1, see www. karger.com/doi/10.1159/000366252). Bidirectional sequencing of the purified PCR products was performed by GATC Biotech (Applied Biosystems ABI 3730xl ${ }^{\mathrm{TM}}$, Konstanz, Germany). Sequences were aligned and compared with reference sequences using the (SeqScape) software v.2.6. 

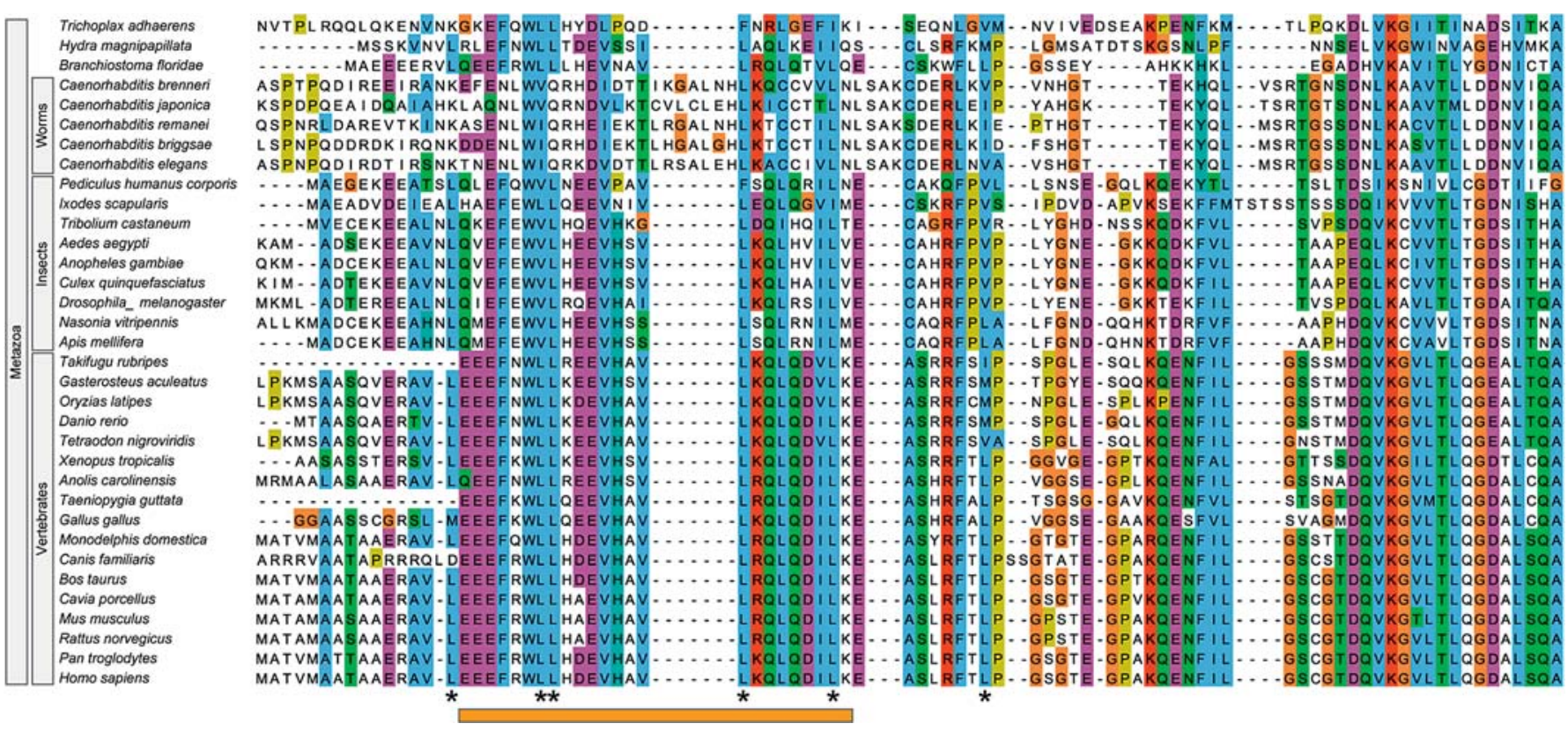

Fig. 3. Multiple sequence alignment. The multiple sequence alignment of the ROGDI protein family amongst metazoans (see Materials and Methods) is shown here. The alignment is displayed using Jalview and colored according to the 'ClustalX' coloring scheme. The proteins are sorted from human to early metazoans from bottom to top, starting from position 1 to position 85 in the human sequence. The 24-aa deletion from position Glu16 to Arg39 is represented by the orange bar. Possible leucines compatible with the Rogdi_lz domain are marked by asterisks.
Sequencing and Mutation Analysis

The Alamut software v.2.2 (Interactive Biosoftware, Rouen, France) was used to collect the analysis of 3 programs used to assess the effect of potential splice variants (Human Splicing Finder, MaxEntScan, NNSplice) [Reese et al., 1997; Yeo and Burge, 2004; Desmet et al., 2009]. dbSNP (build 137, http://www.ncbi.nlm.nih. gov/projects/SNP/) [NCBI Resource Coordinators, 2013] and the Exome Variant Server (NHLBI Exome Sequencing Project (ESP), Seattle, Wash., USA; http://evs.gs.washington.edu/EVS/, March 2013) databases were used to test whether the identified variant was a known variant or polymorphism. The PFAM [Punta et al., 2012] database was used to search for protein domain organization and the eggNOG [Powell et al., 2012] database was used to retrieve the sequences of RODGI orthologs in eukaryotic species (KOG3992). The multiple sequence alignment was extracted from the pre-computed alignments available in eggNOG and further manually refined.

Reverse Transcriptase-PCR and Sequencing Analysis of ROGDI Transcripts

RNA was extracted from fibroblasts of the affected patient (cutaneous biopsy) and 3 unrelated controls by using a RiboPure ${ }^{\mathrm{TM}}$ Kit, followed by DNAse treatment with the TURBO DNA-free ${ }^{\mathrm{TM}}$ Kit (Life Technologies, Carlsbad, Calif., USA). RNA integrity was assessed by gel electrophoresis and RNA concentration by Eppendorf Biophotometer Plus ${ }^{\mathrm{TM}}$ with the Hellma ${ }^{\circledR}$ TrayCell ${ }^{\mathrm{TM}}$ (Eppendorf, Hamburg, Germany). Reverse transcription of $1 \mu \mathrm{g}$ total RNA to cDNA was performed using the iScript ${ }^{\mathrm{TM}} \mathrm{cDNA}$ Synthesis
Kit (BioRad, Hercules, Calif., USA). RT-PCR was performed to determine the exon content of the cDNA from the patient. PCR primers specific for exons 1-3 were designed to amplify the anticipated altered splice site junction (online suppl. table 1). ROGDI cDNA was also partially sequenced to determine the consequence of the genomic mutation on RNA splicing.

\section{Results}

Bidirectional sequencing analysis of ROGDI identified a homozygous mutation c. $117+1 \mathrm{G}>\mathrm{T}$ in the second intron in the proband. This mutation causes a transversion in the canonical splice donor site of intron 2. Segregation analysis validated that each unaffected parent was heterozygous for this mutation (fig. 2E). The mutation was absent from dbSNP and the Exome Variant Server.

The effect of the mutation on the splice site was assessed using 3 different splice site mutation prediction programs (see Materials and Methods). As expected, all programs predicted the destruction of the donor site. The expected consequences were the skipping of exon 2 and an in-frame assembly of exon 3 . The resulting protein is expected to be shorter by 24 amino acids (aa) from posi- 
tion Glu16 to Arg39. Thanks to the PFAM database, the protein could be annotated as containing a leucine zipper domain spanning from position 18 to 277 (domain Rogdi_lz: PF10259). Analysis of the multiple sequence alignment of the protein members of the ROGDI family extracted from eggNOG revealed that the 24 -aa deletion was localized in one of the regions containing a pattern of leucines organized like in leucine zipper domains, which could then affect the function of the protein (fig. 3).

To validate the biological effect of this mutation, we performed RT-PCR analysis and sequencing of the ROGDI cDNA from the proband and unrelated controls. RT-PCR analysis confirmed the absence of exon 2 in the affected patient with a shorter fragment, whereas the control samples yielded a PCR fragment of the expected size (248 bp) (fig. 2F). Sequencing of the RT-PCR products further confirmed the absence of exon 2 in the proband's cDNA and the in-frame assembly of exon 3 (fig. 2G).

\section{Discussion}

The ROGDI gene located on chromosome 16 is composed of 11 coding exons. The encoded protein is 287 aa long and is annotated as a leucine zipper domain-containing protein (UniProt AC: Q9GZN7). We report a novel mutation $c .117+1 \mathrm{G}>\mathrm{T}$ that abrogates the donor splice site in intron 2 and causes the deletion of exon 2 (72 bp) resulting in a shorter protein (24 aa less). This is the first report of a mutation in ROGDI in a sub-Saharan African patient. Our discovery adds to the list of mutations previously identified in this gene in patients with KTZS. Other mutations described in the literature (fig. 1) include deletions, duplications, splicing, and frameshift mutations and are scattered in exons 4-7 and introns 1 and 7 [Schossig et al., 2012a; Mory et al., 2012; Tucci et al., 2013].

The function of ROGDI is yet unknown; however, the protein is present in metazoan species ranging from worms to humans and is highly conserved, suggesting an important functional role (fig. 3).

The loss of 24 aa in the mutant protein likely affects protein function. Indeed, exon 2 contains several highly conserved leucine residues which may contribute to dimerization and DNA binding.

Rare diseases that manifest with neurological symptoms and various dental anomalies have been previously recognized [Bloch-Zupan, 2007]. For example, tuberous sclerosis (OMIM 191100) and Sjögren-Larsson syndrome (OMIM 270200) combine epilepsy and enamel defects among many other features. The function of the ROGDI protein and the link between the dental and brain phenotypes observed in the presence of ROGDI mutations is yet to be understood. Also known as $L z f$, C76152, AU020118 and 0610011C19Rik, ROGDI is transcribed during mouse and human brain development [Jensen et al., 2004]. Rogdi is also expressed in the cap stage of odontogenesis in E14.5 mouse embryos [Laugel-Haushalter et al., 2013]. This suggests that ROGDI is present during and may play a role in brain formation and homeostasis as well as in odontogenesis.

Careful examination of the oral cavity may provide invaluable information for the diagnosis of rare diseases. It facilitated the diagnosis of KTZS in this patient and family, who waited 13 years for a confirmed clinical and molecular diagnosis. Collaborative efforts between reference centers for rare diseases and multidisciplinary health care centers are indispensible for providing an integrative approach to the diagnosis and treatment of patient pathologies. Further research on the role of ROGDI during brain and tooth development as well as in epilepsy and AI is necessary and may allow the discovery of new treatments for KTZS.

\section{Acknowledgement}

We thank the involved family for their participation and contribution. The authors thank Megana Prasad for her careful review of the manuscript. This work was supported by a grant from the University of Strasbourg, the French Ministry of Health (National Program for Clinical Research, PHRC 2008 No. 4266, 'Amelogenesis imperfecta'), the Hôpitaux Universitaires de Strasbourg (API, 2009-2012, 'Development of the oral cavity: from gene to clinical phenotype in human'), IFRO (Institut Français pour la Recherche Odontologique) and EU-funded (ERDF) INTERREG IV/Offensive Sciences Project A27 'Oro-dental manifestations of rare diseases'.

\section{References}

Aldred MJ, Savarirayan R, Crawford PJ: Amelogenesis imperfecta: a classification and catalogue for the 21st century. Oral Dis 9:19-23 (2003).

-Bloch-Zupan A: When neuropediatrics meets odontology. Neuropediatrics 38:57-58 (2007).

-Bloch-Zupan A, Sedano H, Scully C: Dento/Oro/ Craniofacial Anomalies and Genetics, ed 1 (Elsevier, London 2012).

Crawford PJ, Aldred M, Bloch-Zupan A: Amelogenesis imperfecta. Orphanet J Rare Dis 2:17 (2007).

- Desmet FO, Hamroun D, Lalande M, CollodBéroud G, Claustres M, Béroud C: Human Splicing Finder: an online bioinformatics tool to predict splicing signals. Nucleic Acids Res 37:e67 (2009). 
Gadhia K, McDonald S, Arkutu N, Malik K: Amelogenesis imperfecta: an introduction. $\mathrm{Br}$ Dent J 212:377-379 (2012).

Jensen P, Magdaleno S, Lehman KM, Rice DS, Lavallie ER, et al: A neurogenomics approach to gene expression analysis in the developing brain. Brain Res Mol Brain Res 132:116-127 (2004).

Kohlschütter A, Chappuis D, Meier C, Tönz O, Vassella F, Herschkowitz N: Familial epilepsy and yellow teeth: a disease of the CNS associated with enamel hypoplasia. Helv Paediatr Acta 29:283-294 (1974).

Laugel-Haushalter V, Paschaki M, Thibault-Carpentier C, Dembelé D, Dollé P, Bloch-Zupan A: Molars and incisors: show your microarray IDs. BMC Res Notes 6:113 (2013).

-Mory A, Dagan E, Illi B, Duquesnoy P, Mordechai $\mathrm{S}$, et al: A nonsense mutation in the human homolog of Drosophila rogdi causes Kohlschutter-Tonz syndrome. Am J Hum Genet 90:708-714 (2012).
-Mory A, Dagan E, Shahor I, Mandel H, Illi B, et al: Kohlschutter-Tonz syndrome: clinical and genetic insights gained from 16 cases deriving from a close-knit village in northern Israel. Pediatr Neurol 50:421-426 (2014).

NCBI Resource Coordinators: Database resources of the National Center for Biotechnology Information. Nucleic Acids Res 41(Database issue):D8-D20 (2013).

Powell S, Szklarczyk D, Trachana K, Roth A, Kuhn M, et al: eggNOG v3.0: orthologous groups covering 1133 organisms at 41 different taxonomic ranges. Nucleic Acids Res 40(Database issue):D284-D289 (2012).

Punta M, Coggill PC, Eberhardt RY, Mistry J, Tate J, et al: The Pfam protein families database. Nucleic Acids Res 40(Database Issue):D290D301 (2012).
Reese MG, Eeckman FH, Kulp D, Haussler D: Improved splice site detection in Genie. J Comput Biol 4:311-323 (1997).

Schossig A, Wolf NI, Fischer C, Fischer M, Stocker G, et al: Mutations in ROGDI cause Kohlschütter-Tönz syndrome. Am J Hum Genet 90:701-707 (2012a).

Schossig A, Wolf NI, Kapferer I, Kohlschütter A, Zschocke J: Epileptic encephalopathy and amelogenesis imperfecta: Kohlschütter-Tönz syndrome. Eur J Med Genet 55:319-322 (2012b).

Tucci A, Kara E, Schossig A, Wolf NI, Plagnol V, et al: Kohlschütter-Tönz syndrome: mutations in ROGDI and evidence of genetic heterogeneity. Hum Mutat 34:296-300 (2013).

Yeo G, Burge CB: Maximum entropy modeling of short sequence motifs with applications to RNA splicing signals. J Comput Biol 11:377394 (2004). 